\title{
IDENTIFICATION OF THE SPREAD OF BORAX USE IN MEATBALL SKEWERS IN BANTUL DISTRICT, SPECIAL REGION OF YOGYAKARTA
}

\section{Sabtanti Harimurti*), Imelda Horirotul Bariroh, Asep Setiyawan, Ratna Indah Permatasari, Friccillia Dwi Putri, Leni Yasinta Fajriana}

Pharmacy Study Program, Faculty of Medicine and Health Sciences

Universitas Muhammadiyah Yogyakarta, Jalan Barwijaya, Tamantirto, Kasihan, Bantul, Yogyakarta 55183, Indonesia

Received January 20, 2020; Accepted August 20, 2020

\begin{abstract}
Borax is an additive that is often added to some foods such as meatball skewers. The government has banned this ingredient from being added to food because it is harmful to health when consumed for a long time. This study aims to identify the use of borax in Bakso Tusuk or meatball skewers sold in Bantul area. Samples were drawn from 17 sub-districts in Bantul, Special Region of Yogyakarta. Two samples were taken from each sub-district, so the number of total samples was 34. The speed of decay test was carried out by observing the appearance of fungus, maggots, and consistency of the surface of meatball skewers. The presence of borax in the meatball skewers was done by turmeric paper and flame test, while the quantitative analysis utilized the acid-base titration method. Based on the results of the study, all samples tested positive for borax with concentrations between $0.06 \%-5.15 \%$, and the decay rate test showed that the speed of decay was independent of the level of borax.
\end{abstract}

Keywords: borax; identification; meatball skewers.

\section{INTRODUCTION}

Food additives are frequently added during food processing in order to give impressions that the food has a good shape, delicious taste, tempting smell, and is also long-lasting. Constitution has postulated regulation on which food additives are safe to be used in food processing. The goal of using food additives is to make the food favorable, to promote excellent quality, and to ensure its safety to be consumed by society.

Natrium Tetraborat $\left(\mathrm{NaB}_{4} \mathrm{O}_{7} \cdot 10 \mathrm{H}_{2} \mathrm{O}\right)$, or more commonly known as borax, is shaped in white crystal, does not have any smell, and is stable at room temperature. Borax is usually used to produce detergent and antiseptic. When consumed, borax will gradually create sediment and will cumulatively be absorbed by consumers' bodies, which eventually leads to a negative effect (Tubagus et al., 2013).
According to Asteriani et al. (2008), the use of borax in foods must be anticipated, for it may cause a negative effect on the body cumulatively. Consuming 5 grams or more may lead to death for children and infants, while the deadly dosage of consuming borax for adults is 10 to 20 grams (Asteriani et al., 2008).

One of the currently popular and favored foods in our society is Bakso Tusuk or meatball skewers. Not only is it highly affordable, but meatball skewers are also easily found everywhere. The food is highly favored by the community, especially by school-age children, for being sold at a relatively low price and is commonly sold in the school area. The fact that meatball skewers sellers display their food on the street sides should also be a concern. The sellers of meatball skewers usually press the production 
cost by producing meatballs in a large number and by adding preservatives to be able to store the meatballs for more extended periods and, at the same time, make them last longer. Sellers usually use borax as a preservative because it can easily be obtained at an affordable price from shops that sell baking supplies. The use of borax is banned by the Ministry of Health of the Republic of Indonesia through its issued regulation No.772/Menkes/Per/IX/88 and No.1168/Menkes/Per/X/1999.

Regarding the harm of borax added in food, many researchers carried out research on the identification of borax in food. The analysis of qualitative and quantitative was reported. Turmeric paper and flame tests were employed during the qualitative analysis method (Asteriana et al., 2008; Tubagus et al., 2013) while the quantitative one was reported using a spectrophotometer or acid-base titration (Asterina et al., 2008; Tubagus et al., 2013; Aryani et al., 2018, Harimurti et al., 2019). Acid-base titration for borax analysis is very simple without specific instruments required. The preserving nature of borax is also the target of research carried out by observing the resistance of the meatballs to storage time (Wibowo, 2005). Based on the previous explanation, this paper will report the identification of borax in meatball skewers sold in Bantul district to educate the public about the dangers of borax consumption for an extended period.

\section{METHODS}

\section{Instruments and materials}

Instruments used in this research were mortar, stamper, burette (Pyrex®), clamp, stative, measuring pipette (Pyrex®), funnel (Herma $\left.{ }^{\circledR}\right)$, measuring glass (Pyrex $\left.{ }^{\circledR}\right)$, drop pipette, beaker glass (Iwaki Pyrex®), Erlenmeyer (Pyrex ${ }^{\circledR}$ ), volumetric flask (Iwaki Pyrex $\left.{ }^{\circledR}\right)$, porcelain cup, stainless spoon, filter paper, electric stove (Maspion®), digital scale (Mettler Toledo®), flasks, zip-lock plastic bag, and wooden matches.

Materials used in this research were turmeric (Curcuma longa Linn. Syn. Curcuma domestica Val.), all samples of meatball skewers from Bantul district, distilled water (Brataco $\left.{ }^{\circledR}\right), \mathrm{CO}_{2}$-free water, $37 \%$ concentrated hydrochloric acid $(\mathrm{HCl})$ (Brataco®), oxalic acid $\left(\mathrm{H}_{2} \mathrm{C}_{2} \mathrm{H}_{4}\right)$ (Bratacoß), sulfuric acid $\left(\mathrm{H}_{2} \mathrm{SO}_{4}\right)$ (Brataco®), ammonia (Brataco®), sodium hydroxide $(\mathrm{NaOH})($ Brataco $\AA)$, borax, phenolphthalein indicator (pp), methyl orange indicator (mo), and methanol (Brataco®).

\section{Sample}

The samples used in this research were meatball skewers obtained from all sellers in every sub-district of Bantul. The samples were taken by considering a particular area or population, commonly called cluster sampling (Nursalam, 2013).

\section{Sampling technique}

Meatball skewers obtained from the sellers were put inside a previously labeled zip-lock plastic bag. It was then put into a flask containing ice cubes to preserve its condition, and was then kept in a freezer in the research laboratory of UMY. Further, a test to identify the content of borax was done in the research and biochemical laboratory of UMY.

\section{Sample preparation}

The sample of meatball skewers was taken as a whole and was weighed meticulously to measure its weight before being added with $50 \mathrm{ml}$ of $\mathrm{CO}_{2}$-free water. The sample was then mashed using a mortar and stamper and later was filtered using filter paper. The filtrate of the meatball skewers was used in titration test analysis, turmeric test, and flame test. Especially in the flame test, the filtrate must be heated using an electric stove (Maspion ${ }^{\circledR}$ ) until it turned into powder.

The sample used in the decomposition test was one whole meatball skewer, which was rested for three days at room temperature. Its color, smell, and whether or not it collected maggot and fungus, along with its texture, were observed.

\section{Decomposition test}

The meatball skewer was put on a baking paper and was coded. It was left for three days at room temperature and was being observed 
regularly at the same exact time when it was initially prepared (Rahmat et al., 2017).

\section{Flame test}

The samples which had been prepared were then added with five drops of concentrated $\mathrm{H}_{2} \mathrm{SO}_{4}$ and $5 \mathrm{ml}$ of methanol in a porcelain cup and were later burned using wooden matches. Samples that were positive to contain borax would create a green-colored flame (Clarke, 2004; Roth et al., 1988).

\section{Turmeric paper test}

a. The Production of Turmeric Paper

A total of 4 sheets of filter paper were cut into rectangle with a size of $2 \times 4 \mathrm{~cm} .250 \mathrm{~g}$ of turmeric was grated, and its water was filtered. The filter papers were dipped into the turmeric orange water and later were dried. The end product of such a process was called turmeric paper.

b. Qualitative Test using Turmeric Paper

A positive control was made by putting one spoon of borax into a beaker glass (Pyrex®), which was added with distilled water, and was stirred. The solution was dropped onto a turmeric paper. The color created through this process was used as a positive control.

The sample solution was prepared and dropped onto turmeric paper. If it produced the same color as the positive control, the sample was positive to contain borax. The same indication also applied if the sample turned into dark green-blue when being steamed up with ammonia (Roth et al., 1988).

\section{Quantitative analysis}

a. The Standardization of $\mathrm{NaOH}$ Using Oxalic Acid Primary Standard Solution

1) The Preparation of Oxalic Acid

Oxalic acid was used as the primary standard solution. Oxalic acid was made by weighing 0.63 grams of oxalic acid and dissolving it with distilled water inside a volumetric flask until its volume reached 100 $\mathrm{ml}$. Subsequently, $25 \mathrm{ml}$ was taken from the solution and was put into Erlenmeyer.

2) The Preparation of $\mathrm{NaOH}$ solution

Forty grams of $\mathrm{NaOH}$ were weighed accurately, put into a volumetric flask, and was dissolved with $\mathrm{CO}_{2}$-free water until its volume reached $1000 \mathrm{ml}$. $\mathrm{NaOH}$ is a strong base that can quickly react to $\mathrm{CO}_{2}$ so that $\mathrm{CO}_{2}$ free water is required to dissolve it.

3) The Standardization of $\mathrm{NaOH}$ using Oxalic Acid

The prepared oxalic acid solution was added with the phenolphthalein indicator. When the indicator was added, the solution remained clear. However, after being titrated with oxalic acid primary raw solution, the color turned into pink. The titration solution was used as the primary stock solution of $\mathrm{NaOH}$ to standardize $\mathrm{HCl}$, which would be used to measure the concentration of borax in meatball skewers in Bantul district.

b. The Preparation of $\mathrm{HCl}$ for Titration

The titration process used HCI because $\mathrm{HCl}$ can create salt that is easily dissolved in water (Day et al., 1996).

1) The Preparation of $\mathrm{HCl}$

As much as $9.90 \mathrm{ml}$ of concentrated $\mathrm{HCl}$ $37 \%$ was dissolved in distilled water until it reached $1000 \mathrm{ml}$ in a volumetric flask to prepare $1.1 \mathrm{M}$ of $\mathrm{HCl}$.

c. The Standardization of $\mathrm{HCl}$

Twenty five $\mathrm{ml}$ of a secondary standard solution of $\mathrm{HCl}$ was put into Erlenmeyer and was then added with phenolphthalein indicator. It was later being titrated with a primary stock solution of $\mathrm{NaOH} 1 \mathrm{~N}$, which had been standardized with oxalic acid. Titration was stopped right after the solution turned into pink, and the volume was recorded. Replication was done three times. The HCI normality was then measured using the following equation 1 .

$$
\mathrm{V}_{1} \cdot \mathrm{N}_{1}=\mathrm{V}_{2} \cdot \mathrm{N}_{2}
$$

with the following details;

$\mathrm{V}_{1}: \mathrm{NaOH}$ volume

$\mathrm{N}_{1}$ : $\mathrm{NaOH}$ normality

$\mathrm{V}_{2}: \mathrm{HCl}$ volume

$\mathrm{N}_{2}: \mathrm{HCl}$ normality 
d. The Measurement of Borax Concentration on the Sample

Ten $\mathrm{ml}$ was taken from the sample solution and was then put into Erlenmeyer. It was further added with five drops of methyl orange indicator and was titrated with $\mathrm{HCl}$ until the color changed into pink. The titration test was replicated three times. The average volume of titration used in each of the samples was measured. Afterward, the concentration of the borax was measured using the following equation 2 .

Borax

Concentration $(\%)$

$$
=\frac{5 x V x N x B E \text { quivalent weight }}{\text { Sample weight }(\mathrm{mg})} \times 100 \%(2)
$$

with the following details:

V: Sample volume

$\mathrm{N}$ : $\mathrm{HCl}$ normality

BE: Equivalent weight

\section{RESULTS AND DISCUSSION}

Qualitative analysis of borax content in meatball Skewers in Bantul district

In this study, qualitative analysis was carried out using three methods, namely decomposition test, turmeric paper test, and flame test. Particularly in this research, the decay test was carried out to study the speed of spoilage of the meatballs considering that borax is often added for preservative purposes. Observations were accomplished by observing the changes that occurred over time. The changes observed were changes in color and odor, the presence or absence of maggots, and the presence or absence of mold. Two qualitative methods that were implemented were the turmeric paper test and flame test. Both tests were carried out to identify the initial presence of borax in the meatball skewer. Two kinds of the test were carried out to confirm each other.

a. Decomposition test results

The decomposition test results, as shown in table 1 , indicate that the average samples experienced significant changes on the second day, but 14 samples with sample codes of BT $02,09,10,11,15,17,18,19,20,27,30,31$, and 33 indicate rather significant changes on the first day. All samples experienced considerable transformations, turning brownish, black beige, or black, producing a bad smell and growing fungi.

Table 1. The qualitative inspection results of Borax in Bakso Tusuk in Bantul district

\begin{tabular}{|c|c|c|c|c|c|c|}
\hline \multirow{2}{*}{ Sample } & \multirow{2}{*}{$\begin{array}{c}\text { Sample } \\
\text { Code }\end{array}$} & \multicolumn{3}{|c|}{ Decay Test } & \multirow{2}{*}{$\begin{array}{c}\text { Turmeric } \\
\text { Test }\end{array}$} & \multirow{2}{*}{$\begin{array}{c}\text { Flame } \\
\text { Test }\end{array}$} \\
\hline & & Day 1 & Day 2 & Day 3 & & \\
\hline $\begin{array}{l}\text { Bambang } \\
\text { lipuro } 1\end{array}$ & BT.01 & $\begin{array}{l}\text { Brownish beige, } \\
\text { slightly wet, } \\
\text { maggots -, fungi - }\end{array}$ & $\begin{array}{l}\text { Brownish beige, } \\
\text { undamaged, } \\
\text { maggots -, fungi - } \\
\text {, smelly }\end{array}$ & $\begin{array}{l}\text { Brownish beige, } \\
\text { undamaged, } \\
\text { maggots -, fungi -, } \\
\text { smelly }\end{array}$ & + & + \\
\hline $\begin{array}{l}\text { Bambang } \\
\text { lipuro } 2\end{array}$ & BT.02 & $\begin{array}{l}\text { Brownish, } \\
\text { undamaged, } \\
\text { maggot -, fungi + }\end{array}$ & $\begin{array}{l}\text { Color began to } \\
\text { turn black, } \\
\text { watery, maggots - } \\
\text {, fungi ++ }\end{array}$ & $\begin{array}{l}\text { Blackish, watery, } \\
\text { soft, maggots -, } \\
\text { fungi ++++, } \\
\text { yellow-colored } \\
\text { fungi, bad odor }\end{array}$ & + & + \\
\hline $\begin{array}{l}\text { Bangunta } \\
\text { pan } 1\end{array}$ & BT.03 & $\begin{array}{l}\text { Brownish beige, } \\
\text { undamaged, } \\
\text { maggots -, fungi - }\end{array}$ & $\begin{array}{l}\text { Brownish beige, } \\
\text { dry, hard, ant }+ \text {, } \\
\text { maggot -, fungi - }\end{array}$ & $\begin{array}{l}\text { Dark brown, dry, } \\
\text { hard, maggots -, } \\
\text { fungi }+, \text { smelly }\end{array}$ & + & + \\
\hline $\begin{array}{l}\text { Bangunta } \\
\text { pan } 2\end{array}$ & BT.04 & $\begin{array}{l}\text { Brown beige, } \\
\text { undamaged, } \\
\text { maggots -, fungi - }\end{array}$ & $\begin{array}{l}\text { Brownish, dry, } \\
\text { hard, maggots -, } \\
\text { fungi - }\end{array}$ & $\begin{array}{l}\text { Color began to turn } \\
\text { black, dry, hard, } \\
\text { maggots -, fungi }+ \text {, } \\
\text { bad odor }\end{array}$ & + & + \\
\hline Bantul 1 & BT.05 & $\begin{array}{l}\text { Beige, maggots -, } \\
\text { fungi - }\end{array}$ & $\begin{array}{l}\text { Brownish beige, } \\
\text { undamaged, } \\
\text { maggots -, fungi - } \\
\text {, smelly }\end{array}$ & $\begin{array}{l}\text { Brownish beige, } \\
\text { undamaged, } \\
\text { maggots -, fungi -, } \\
\text { smelly }\end{array}$ & + & + \\
\hline
\end{tabular}




\begin{tabular}{|c|c|c|c|c|c|c|}
\hline Bantul 2 & BT.06 & $\begin{array}{l}\text { Beige, ants }+, \\
\text { maggots -, fungi } \\
\text { - }\end{array}$ & $\begin{array}{l}\text { Brownish beige, } \\
\text { undamaged, } \\
\text { slightly watery, } \\
\text { maggots -, } \\
\text { fungi -, smelly }\end{array}$ & $\begin{array}{l}\text { Brownish beige, } \\
\text { undamaged, } \\
\text { watery, maggots } \\
\text {-, fungi -, bad } \\
\text { odor }\end{array}$ & + & + \\
\hline Dlingo 1 & BT.07 & $\begin{array}{l}\text { Brownish beige, } \\
\text { undamaged, ants } \\
++++, \text { maggots - } \\
\text {, fungi - }\end{array}$ & $\begin{array}{l}\text { Dark brown, } \\
\text { hard, ants }+++, \\
\text { maggots -, } \\
\text { fungi - }\end{array}$ & $\begin{array}{l}\text { Dark brown, } \\
\text { hard, ants +++, } \\
\text { maggots -, fungi } \\
\text {-, bad odor }\end{array}$ & + & + \\
\hline Dlingo 2 & BT.08 & $\begin{array}{l}\text { Dark brown, } \\
\text { undamaged, } \\
\text { maggots -, fungi } \\
\text { - }\end{array}$ & $\begin{array}{l}\text { Grayish, } \\
\text { hard, ants +, } \\
\text { maggots -, } \\
\text { fungi - }\end{array}$ & $\begin{array}{l}\text { Color began to } \\
\text { turn black, hard, } \\
\text { ants }++, \text { maggots } \\
-, \text { fungi -, began } \\
\text { to damage }\end{array}$ & + & + \\
\hline Imogiri 1 & ВТ.09 & $\begin{array}{l}\text { Brownish beige, } \\
\text { undamaged, ants } \\
+, \text { maggot }- \\
\text { fungi }+\end{array}$ & $\begin{array}{l}\text { Brownish beige, } \\
\text { ants }+, \text { maggots } \\
-, \text { fungi }++, \\
\text { smelly }\end{array}$ & $\begin{array}{l}\text { Brownish beige, } \\
\text { undamaged, } \\
\text { maggots -, fungi } \\
+++, \text { bad odor }\end{array}$ & + & $\mathrm{TD}$ \\
\hline Imogiri 2 & BT.10 & $\begin{array}{l}\text { Brownish beige, } \\
\text { undamaged, } \\
\text { maggot -, fungi } \\
+\end{array}$ & $\begin{array}{l}\text { Color began to } \\
\text { turn black, } \\
\text { partly dry, } \\
\text { maggots -, } \\
\text { fungi }+, \text { smelly }\end{array}$ & $\begin{array}{l}\text { Black, hard, dry, } \\
\text { maggots -, fungi } \\
++, \text { bad odor }\end{array}$ & + & + \\
\hline Jetis 1 & BT.11 & $\begin{array}{l}\text { Brownish beige, } \\
\text { undamaged, } \\
\text { maggot -, fungi } \\
+\end{array}$ & $\begin{array}{l}\text { Color began to } \\
\text { turn black, } \\
\text { rather soft, } \\
\text { maggots -, } \\
\text { fungi }+ \text {, smelly }\end{array}$ & $\begin{array}{l}\text { Blackish, soft, } \\
\text { watery, maggots } \\
\text {-, fungi }+++, \text { bad } \\
\text { odor }\end{array}$ & + & + \\
\hline Jetis 2 & BT.12 & $\begin{array}{l}\text { Brownish beige, } \\
\text { undamaged, } \\
\text { maggots -, fungi } \\
\text { - }\end{array}$ & $\begin{array}{l}\text { Brown, slightly } \\
\text { hard, maggots -, } \\
\text { fungi + }\end{array}$ & $\begin{array}{l}\text { Blackish brown, } \\
\text { rather hard, } \\
\text { maggots -, fungi } \\
++, \text { bad odor }\end{array}$ & + & TD \\
\hline Kasihan 1 & BT.13 & $\begin{array}{l}\text { Creamy-colored, } \\
\text { undamaged, } \\
\text { maggots -, fungi } \\
\text { - }\end{array}$ & $\begin{array}{l}\text { Grayish beige, } \\
\text { rather dry, } \\
\text { slightly hard, } \\
\text { maggots -, } \\
\text { fungi - }\end{array}$ & $\begin{array}{l}\text { Grayish, dry, } \\
\text { hard, maggots -- } \\
\text { fungi -, bad odor }\end{array}$ & + & $\mathrm{TD}$ \\
\hline Kasihan 2 & BT.14 & $\begin{array}{l}\text { Brownish beige, } \\
\text { maggots -, fungi } \\
\text { - }\end{array}$ & $\begin{array}{l}\text { Grayish brown, } \\
\text { maggots -, } \\
\text { fungi -, smelly }\end{array}$ & $\begin{array}{l}\text { Grayish, } \\
\text { undamaged, } \\
\text { maggots -, fungi } \\
\text {-, bad odor }\end{array}$ & + & + \\
\hline Kretek 1 & BT.15 & $\begin{array}{l}\text { Brownish beige, } \\
\text { maggots -, fungi } \\
+\end{array}$ & $\begin{array}{l}\text { Dark brown, } \\
\text { soft, maggots, } \\
\text { fungi ++, } \\
\text { smelly }\end{array}$ & $\begin{array}{l}\text { Blackish brown, } \\
\text { soft, maggots, } \\
\text { fungi }++ \text {, bad } \\
\text { odor }\end{array}$ & + & + \\
\hline Kretek 2 & BT.16 & $\begin{array}{l}\text { Brownish beige, } \\
\text { maggots -, fungi } \\
\text { - }\end{array}$ & $\begin{array}{l}\text { Dark brown, } \\
\text { soft, maggots, } \\
\text { fungi -, smelly }\end{array}$ & $\begin{array}{l}\text { Blackish brown, } \\
\text { soft, maggots - } \\
\text {, fungi -, bad } \\
\text { odor }\end{array}$ & + & + \\
\hline Pajangan 1 & BT.17 & $\begin{array}{l}\text { Brown, maggots } \\
-, \text { fungi }+\end{array}$ & $\begin{array}{l}\text { Dark brown, } \\
\text { maggots -, } \\
\text { fungi }+, \text { smell }\end{array}$ & $\begin{array}{l}\text { Blackish, } \\
\text { undamaged, } \\
\text { maggots -, fungi } \\
++, \text { bad odor }\end{array}$ & + & TD \\
\hline Pajangan 2 & BT.18 & $\begin{array}{l}\text { Brown beige, } \\
\text { ants }+, \text { maggots } \\
-, \text { fungi }+\end{array}$ & $\begin{array}{l}\text { Brown, } \\
\text { undamaged, } \\
\text { maggots -, } \\
\text { fungi }+, \text { smelly }\end{array}$ & $\begin{array}{l}\text { Dark brown, } \\
\text { undamaged, } \\
\text { maggots -, fungi } \\
++, \text { smelly }\end{array}$ & + & TD \\
\hline
\end{tabular}




\begin{tabular}{|c|c|c|c|c|c|c|}
\hline Pandak 1 & BT.19 & $\begin{array}{l}\text { Brownish beige, } \\
\text { undamaged, } \\
\text { maggots -, fungi } \\
+\end{array}$ & $\begin{array}{l}\text { Gray, slightly } \\
\text { hard, maggots -, } \\
\text { fungi }+ \text {, smelly }\end{array}$ & $\begin{array}{l}\text { Gray, hard, } \\
\text { maggots -, fungi } \\
++, \text { smelly }\end{array}$ & + & + \\
\hline Pandak 2 & BT.20 & $\begin{array}{l}\text { Brownish beige, } \\
\text { undamaged, } \\
\text { maggot -, fungi } \\
+\end{array}$ & $\begin{array}{l}\text { Dark brown, } \\
\text { hard, maggots -, } \\
\text { fungi }+, \text { smelly }\end{array}$ & $\begin{array}{l}\text { Blackish brown, } \\
\text { hard, maggots -, } \\
\text { fungi }++ \text {, bad } \\
\text { odor }\end{array}$ & + & + \\
\hline Pleret 1 & BT.21 & $\begin{array}{l}\text { Grayish, } \\
\text { undamaged, ants } \\
+, \text { maggots -, } \\
\text { fungi - }\end{array}$ & $\begin{array}{l}\text { Grayish, hard, } \\
\text { ants }+, \text { maggots } \\
\text {-, fungi - }\end{array}$ & $\begin{array}{l}\text { Blackish, dry, } \\
\text { hard, maggots -, } \\
\text { fungi +, bad odor }\end{array}$ & + & TD \\
\hline Pleret 2 & BT.22 & $\begin{array}{l}\text { Blackish beige, } \\
\text { undamaged, } \\
\text { maggots -, fungi } \\
\text { - }\end{array}$ & $\begin{array}{l}\text { Grayish, hard, } \\
\text { maggots -, } \\
\text { fungi - }\end{array}$ & $\begin{array}{l}\text { Gray, dry, hard, } \\
\text { maggots -, fungi } \\
\text {-, smelly }\end{array}$ & + & TD \\
\hline Piyungan 1 & BT.23 & $\begin{array}{l}\text { Blackish brown, } \\
\text { undamaged, } \\
\text { maggots -, fungi } \\
\text { - }\end{array}$ & $\begin{array}{l}\text { Blackish brown, } \\
\text { hard, ants }+ \text {, } \\
\text { maggot -, fungi } \\
\text { - }\end{array}$ & $\begin{array}{l}\text { Blackish, dry, } \\
\text { hard, maggots -, } \\
\text { fungi -, smelly }\end{array}$ & + & + \\
\hline Piyungan 2 & BT.24 & $\begin{array}{l}\text { Brownish beige, } \\
\text { undamaged, } \\
\text { maggots -, fungi } \\
\text { - }\end{array}$ & $\begin{array}{l}\text { Brown, hard, } \\
\text { dry, ants +, } \\
\text { maggots -, } \\
\text { fungi - }\end{array}$ & $\begin{array}{l}\text { Dark brown, dry, } \\
\text { hard, maggots -, } \\
\text { fungi }+, \text { smelly }\end{array}$ & + & + \\
\hline Pundong 1 & BT.25 & $\begin{array}{l}\text { Brownish beige, } \\
\text { undamaged, } \\
\text { maggots -, fungi } \\
\text { - }\end{array}$ & $\begin{array}{l}\text { Brown, slightly } \\
\text { soft, maggots -, } \\
\text { fungi -, smelly }\end{array}$ & $\begin{array}{l}\text { Dark brown, } \\
\text { soft, maggots - } \\
\text {, fungi -, bad } \\
\text { odor }\end{array}$ & + & + \\
\hline Pundong 2 & BT.26 & $\begin{array}{l}\text { Brownish beige, } \\
\text { undamaged, } \\
\text { maggots -, fungi } \\
\text { - }\end{array}$ & $\begin{array}{l}\text { Brown color, } \\
\text { slightly soft, } \\
\text { maggots -, } \\
\text { fungi -, smelly }\end{array}$ & $\begin{array}{l}\text { Dark brown, } \\
\text { soft, maggots -, } \\
\text { fungi -, bad odor }\end{array}$ & + & + \\
\hline Sanden 1 & BT.27 & $\begin{array}{l}\text { Brownish beige, } \\
\text { maggots -, fungi } \\
+\end{array}$ & $\begin{array}{l}\text { Blackish brown, } \\
\text { undamaged, } \\
\text { maggots -, } \\
\text { fungi }+, \text { smelly }\end{array}$ & $\begin{array}{l}\text { Blackish, } \\
\text { undamaged, } \\
\text { maggots -, fungi } \\
+, \text { bad odor }\end{array}$ & + & + \\
\hline Sanden 2 & BT.28 & $\begin{array}{l}\text { White to beige, } \\
\text { undamaged, } \\
\text { maggots -, fungi } \\
\text { - }\end{array}$ & $\begin{array}{l}\text { Light beige, } \\
\text { slightly hard, } \\
\text { slightly dry, } \\
\text { maggots -, } \\
\text { fungi }+ \text {, smelly }\end{array}$ & $\begin{array}{l}\text { Beige, hard, dry, } \\
\text { maggots -, fungi } \\
+, \text { bad odor }\end{array}$ & + & + \\
\hline Sedayu 1 & BT.29 & $\begin{array}{l}\text { Brownish beige, } \\
\text { undamaged, } \\
\text { maggots -, fungi } \\
+\end{array}$ & $\begin{array}{l}\text { Blackish brown, } \\
\text { slightly soft, } \\
\text { maggots -, } \\
\text { fungi ++, } \\
\text { smelly }\end{array}$ & $\begin{array}{l}\text { Blackish, watery, } \\
\text { soft, maggots -, } \\
\text { fungi }+++ \text {, bad } \\
\text { odor }\end{array}$ & + & + \\
\hline Sedayu 2 & BT.30 & $\begin{array}{l}\text { Brownish beige, } \\
\text { undamaged, } \\
\text { maggots -, fungi } \\
+\end{array}$ & $\begin{array}{l}\text { Blackish brown, } \\
\text { slightly soft, } \\
\text { maggots -, } \\
\text { fungi }+, \text { smelly }\end{array}$ & $\begin{array}{l}\text { Blackish, watery, } \\
\text { soft, maggots -, } \\
\text { fungi }+ \text {, bad odor }\end{array}$ & + & + \\
\hline Sewon 1 & BT.31 & $\begin{array}{l}\text { Brownish beige, } \\
\text { undamaged, } \\
\text { maggots -, fungi } \\
+\end{array}$ & $\begin{array}{l}\text { Blackish brown, } \\
\text { slightly soft, } \\
\text { maggots -, } \\
\text { fungi }+, \text { smelly }\end{array}$ & $\begin{array}{l}\text { Blackish brown, } \\
\text { soft, watery, } \\
\text { maggots -, fungi } \\
+, \text { bad odor }\end{array}$ & + & + \\
\hline Sewon 2 & BT.32 & $\begin{array}{l}\text { Brownish beige, } \\
\text { undamaged, } \\
\text { maggots -, fungi } \\
\text { - }\end{array}$ & $\begin{array}{l}\text { Blackish brown, } \\
\text { soft, maggots -, } \\
\text { fungi -, smelly }\end{array}$ & $\begin{array}{l}\text { Blackish, soft, } \\
\text { maggots -, fungi } \\
\text {-, bad odor }\end{array}$ & + & + \\
\hline
\end{tabular}




\begin{tabular}{|c|c|c|c|c|c|c|}
\hline Srandakan 1 & BT.33 & $\begin{array}{l}\text { Brownish beige, } \\
\text { dry and hard, } \\
\text { maggots -, fungi } \\
+\end{array}$ & $\begin{array}{l}\text { Blackish brown, } \\
\text { dry, } \\
\text { hard, maggots -, } \\
\text { fungi +, smelly }\end{array}$ & $\begin{array}{l}\text { Blackish, dry, } \\
\text { hard, maggots - } \\
\text {, fungi +, bad } \\
\text { odor }\end{array}$ & + & $\mathrm{TD}$ \\
\hline Srandakan 2 & BT.34 & $\begin{array}{l}\text { Brown, maggots } \\
- \text {, fungi - }\end{array}$ & $\begin{array}{l}\text { Dark brown, } \\
\text { maggots -, } \\
\text { fungi - }\end{array}$ & $\begin{array}{l}\text { Dark brown, } \\
\text { undamaged, } \\
\text { maggots -, fungi } \\
\text {-, smelly }\end{array}$ & + & + \\
\hline
\end{tabular}

Source: Primary Data 2016-2017

\section{Information:}

Decay Test

- $\quad$ = No Fungi, Maggots

$+\quad=$ No Fungi Detected

$++=1 / 2$ Funged Surface

$+++=3 / 4$ Funged Surface

\section{Turmeric Paper Test}

- $\quad=$ Negative against the Turmeric Paper Test

$+\quad=$ Positive against the Turmeric Paper Test

According to Wibowo (2005), the factors that influence the decomposition of meatball skewers are the various amount and kinds of meat, and the preservative added, which cannot strengthen the researcher' hypothesis so that another more specific testing to analyze borax content in the samples needs to be conducted.

b. Turmeric paper test results

The results of the turmeric paper test for meatball skewers samples are presented in table 1. The findings indicate that all 34 meatball skewers samples positively contained

\section{Flame Test}

$+\quad=$ Positive on the Flame Test

$\mathrm{TD}=$ No Flame Test Detected borax since they showed the same color as the positive control. The samples were stated to be positive if they showed the same color as the positive control, and they were stated to be negative if the color was the same as the negative control. The principle of this test is that the turmeric extract attached to the filter paper contains curcumin. Curcumin has two forms of tautomer, namely ketone, and enol. The ketone and hydroxyl groups interact with boric acid to produce a red compound called rososianin. The curcumin and borate complex can be seen in figure 1 .

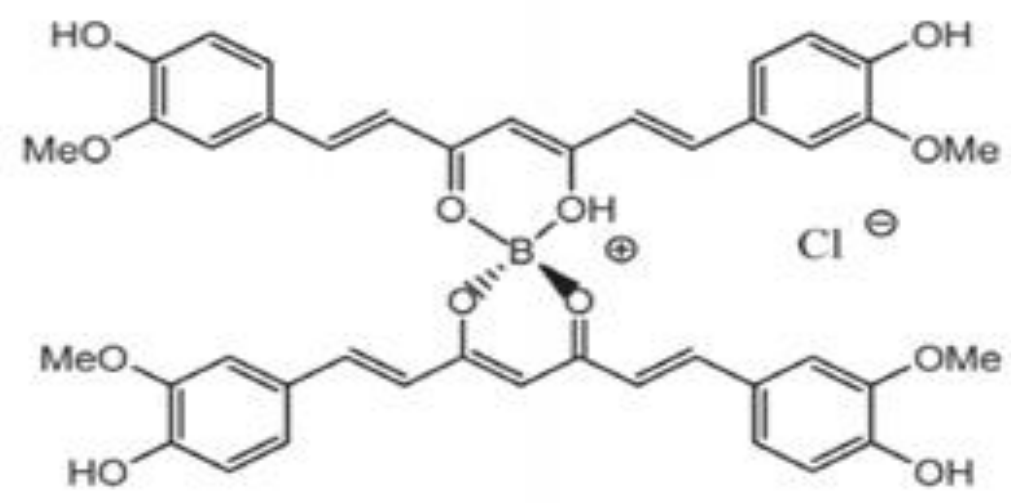

Figure 1. Curcumin complex with borate forming rososianin (Grynkiewicz et al., 2012) 
c. Flame test result

The research result (Table 1) using flame indicates that 8 out of 34 samples were not detected for borax content, and the 26 samples were detected for borax content. This is shown from the same color between the flame and the positive control. The samples that were not detected by green flame were caused by several factors such as the existence of obstructing compounds in meatball skewers samples that generated yellowish-red blue flame. This was because the samples did not go through the process of removing obstructing compounds during the pollination process.

\section{Quantitative analysis with acid-base titration}

The titration volume was known from the titrated samples. The content calculation was conducted, and its result is presented in table 2. The result of the titration indicates that all samples positively contained borax with various dosages. The average number of the samples was calculated, added, and deduced for the highest and lowest content of meatball skewers in Bantul District, Special Region of Yogyakarta. The highest content of borax in meatball skewers in Bantul District, Special Region of Yogyakarta was 5.15\% (Pandak 2), the lowest content was $0.06 \%$ (Banguntapan 2 ), and the average content was $1.64 \%$. This research indicates that all samples contained borax with various amounts.

Table 2. The results of acid-based titration test of borax in Meatball Skewers samples in Bantul district.

\begin{tabular}{|c|c|c|c|c|c|c|c|}
\hline \multirow{2}{*}{ Sample } & \multirow{2}{*}{$\begin{array}{l}\text { Sample } \\
\text { Code }\end{array}$} & \multirow{2}{*}{$\begin{array}{c}\text { Weight of } 1 \\
\text { ball of } \\
\text { meatball } \\
\text { skewers (g) }\end{array}$} & \multicolumn{3}{|c|}{$\mathrm{HCl}(\mathrm{ml})$} & \multirow{2}{*}{$\begin{array}{l}\text { Average } \mathbf{H C l} \\
(\mathrm{ml})\end{array}$} & \multirow{2}{*}{$\begin{array}{c}\text { Borax } \\
\text { content } \\
(\%) \\
\end{array}$} \\
\hline & & & $\begin{array}{c}\text { Rep. } \\
\text { I }\end{array}$ & $\begin{array}{l}\text { Rep. } \\
\text { II }\end{array}$ & $\begin{array}{l}\text { Rep. } \\
\text { III }\end{array}$ & & \\
\hline Bambanglipuro 1 & ВТ.01 & 10.684 & 9.40 & 9.10 & 9.10 & $9.20 \pm 0.14$ & $3.53 \pm 0.05$ \\
\hline Bambanglipuro 2 & BТ.02 & 13.249 & 6.30 & 6.10 & 6.40 & $6.27 \pm 0.12$ & $1.94 \pm 0.04$ \\
\hline Banguntapan 1 & BT.03 & 13.968 & 7.70 & 7.60 & 7.40 & $7.57 \pm 0.12$ & $2.22 \pm 0.04$ \\
\hline Banguntapan 2 & BT.04 & 4.683 & 0.20 & 0.00 & 0.00 & $0.07 \pm 0.09$ & $0.06 \pm 0.08$ \\
\hline Bantul 1 & BT.05 & 10.522 & 3.60 & 3.50 & 3.40 & $3.50 \pm 0.08$ & $1.36 \pm 0.03$ \\
\hline Bantul 2 & BТ.06 & 4.642 & 1.30 & 1.40 & 1.50 & $1.40 \pm 0.08$ & $1.24 \pm 0.07$ \\
\hline Dlingo 1 & BT.07 & 14.744 & 0.90 & 0.70 & 0.60 & $0.73 \pm 0.12$ & $0.20 \pm 0.03$ \\
\hline Dlingo 2 & ВТ.08 & 4.888 & 1.20 & 1.00 & 1.20 & $1.13 \pm 0.09$ & $0.95 \pm 0.08$ \\
\hline Imogiri 1 & ВТ.09 & 10.057 & 2.70 & 2.70 & 2.70 & $2.70 \pm 0.00$ & $1.10 \pm 0.00$ \\
\hline Imogiri 2 & BT.10 & 13.39 & 6.70 & 6.60 & 6.50 & $6.60 \pm 0.08$ & $2.02 \pm 0.02$ \\
\hline Jetis 1 & BT.11 & 16.106 & 4.00 & 4.00 & 4.00 & $4.00 \pm 0.00$ & $1.02 \pm 0.00$ \\
\hline Jetis 2 & BТ.12 & 9.569 & 3.60 & 3.90 & 3.70 & $3.73 \pm 0.12$ & $1.59 \pm 0.05$ \\
\hline Kasihan 1 & BT.13 & 3.747 & 2.00 & 1.80 & 2.10 & $1.97 \pm 0.12$ & $2.15 \pm 0.15$ \\
\hline Kasihan 2 & BТ.14 & 9.562 & 4.30 & 4.00 & 3.90 & $4.07 \pm 0.17$ & $1.74 \pm 0.07$ \\
\hline Kretek 1 & BT.15 & 11.527 & 7.10 & 6.80 & 6.90 & $6.93 \pm 0.12$ & $2.46 \pm 0.04$ \\
\hline Kretek 2 & BT.16 & 4.956 & 9.40 & 9.10 & 9.10 & $4.97 \pm 0.12$ & $4.11 \pm 0.10$ \\
\hline Pajangan 1 & BT.17 & 11.493 & 6.30 & 6.10 & 6.40 & $2.63 \pm 0.09$ & $0.94 \pm 0.03$ \\
\hline Pajangan 2 & BT.18 & 10.862 & 7.70 & 7.60 & 7.40 & $3.53 \pm 0.12$ & $1.33 \pm 0.05$ \\
\hline Pandak 1 & ВТ.19 & 17.444 & 0.20 & 0.00 & 0.00 & $4.37 \pm 0.09$ & $1.03 \pm 0.02$ \\
\hline Pandak 2 & BT.20 & 3.476 & 3.60 & 3.50 & 3.40 & $4.37 \pm 0.19$ & $5.15 \pm 0.22$ \\
\hline Pleret 1 & BT.21 & 18.783 & 1.30 & 1.40 & 1.50 & $3.03 \pm 0.05$ & $0.66 \pm 0.01$ \\
\hline Pleret 2 & BT.22 & 7.111 & 0.90 & 0.70 & 0.60 & $2.20 \pm 0.14$ & $1.27 \pm 0.08$ \\
\hline Piyungan 1 & BT.23 & 6.181 & 1.20 & 1.00 & 1.20 & $4.10 \pm 0.16$ & $2.72 \pm 0.11$ \\
\hline Piyungan 2 & BT.24 & 22.681 & 2.70 & 2.70 & 2.70 & $3.90 \pm 0.21$ & $0.70 \pm 0.04$ \\
\hline Pundong 1 & BT.25 & 9.754 & 6.70 & 6.60 & 6.50 & $4.20 \pm 0.16$ & $1.76 \pm 0.07$ \\
\hline Pundong 2 & BT.26 & 10.203 & 4.00 & 4.00 & 4.00 & $3.80 \pm 0.14$ & $1.53 \pm 0.06$ \\
\hline Sanden 1 & BT.27 & 10.702 & 3.60 & 3.90 & 3.70 & $3.47 \pm 0.12$ & $1.32 \pm 0.05$ \\
\hline Sanden 2 & BT.28 & 3.852 & 2.00 & 1.80 & 2.10 & $2.67 \pm 0.12$ & $2.84 \pm 0.13$ \\
\hline
\end{tabular}




\begin{tabular}{lccccccc}
\hline Sedayu 1 & BT.29 & 17.329 & 4.30 & 4.00 & 3.90 & $3.43 \pm 0.17$ & $0.91 \pm 0.04$ \\
\hline Sedayu 2 & BT.30 & 7.602 & 7.10 & 6.80 & 6.90 & $1.87 \pm 0.19$ & $0.10 \pm 0.01$ \\
\hline Sewon 1 & BT.31 & 9.821 & 9.40 & 9.10 & 9.10 & $4.27 \pm 0.21$ & $1.83 \pm 0.09$ \\
\hline Sewon 2 & BT.32 & 14.666 & 6.30 & 6.10 & 6.40 & $4.30 \pm 0.16$ & $1.19 \pm 0.05$ \\
\hline Srandakan 1 & BT.33 & 13.399 & 7.70 & 7.60 & 7.40 & $3.17 \pm 0.09$ & $0.97 \pm 0.03$ \\
\hline Srandakan 2 & BT.34 & 15.612 & 7.70 & 7.80 & 7.80 & $7.77 \pm 0.05$ & $2.04 \pm 0.01$ \\
\hline Total & & & & & & 55.76 \\
\hline Average & & & & & & & 1.64 \\
\hline Highest value & & & & & & 0.06 \\
\hline Lowest value & & & & & & & \\
\hline
\end{tabular}

Source: Primary Data 2016-2017

Map borax distribution in meatball Skewers in Bantul district

Bantul District is a district with 50,685 $\mathrm{Ha}$ in width and has 17 sub-districts namely Bambanglipuro, Banguntapan, Bantul, Dlingo, Imogiri, Jetis, Kasihan, Kretek, Pajangan, Pandak, Pleret, Piyungan, Pundong, Sanden,
Sedayu, Sewon, and Srandakan (BPS Kabupaten Bantul, 2015). Figure 1 below illustrates that the samples from all subdistricts positively contained borax with the highest content of $5.15 \%$ (Pandak 2), the lowest content of $0.06 \%$ (Banguntapan 2), and average content of $1.64 \%$.

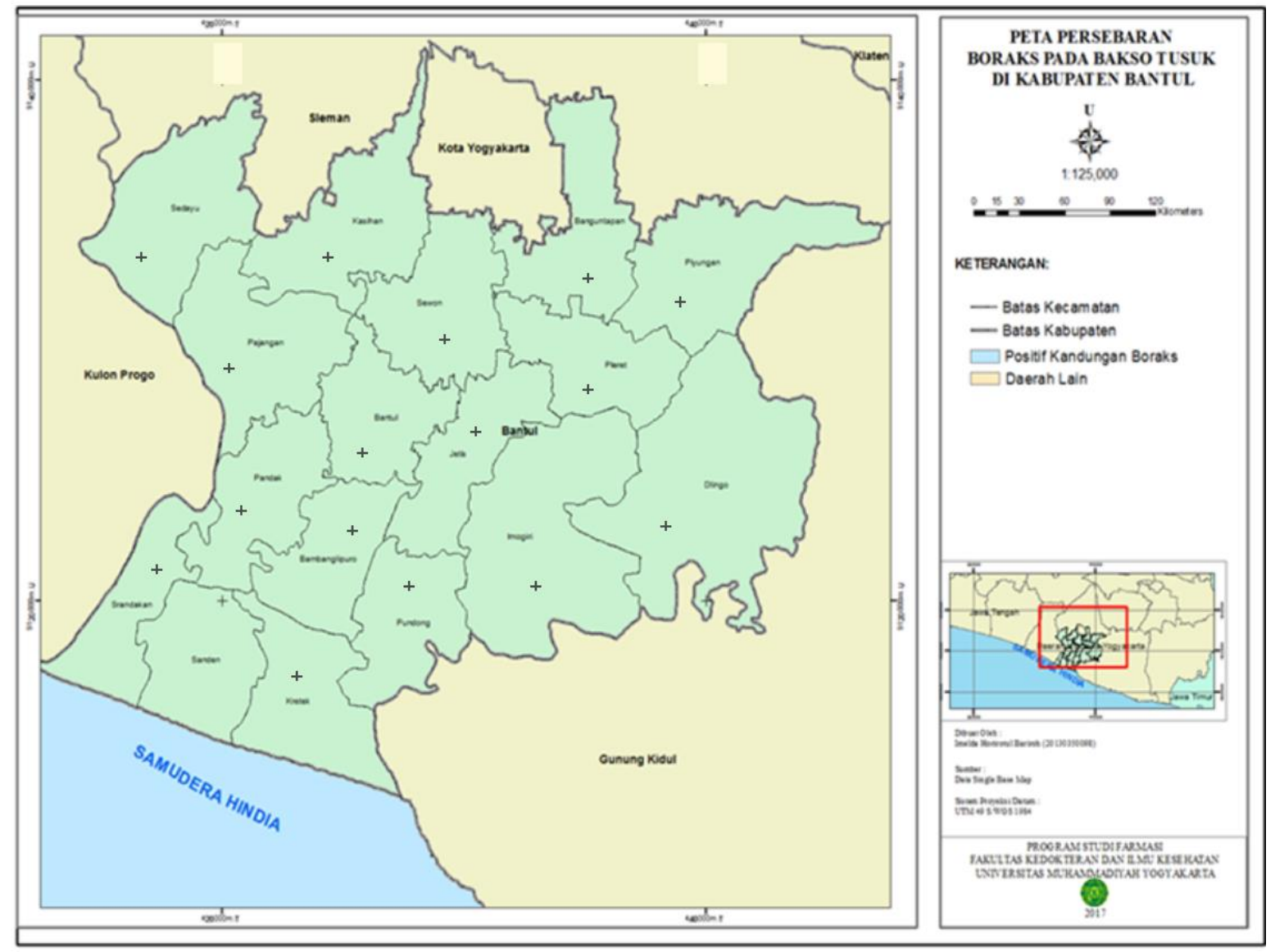

Figure 2. The map of distribution of meatball Skewers sample points containing borax in Bantul district (Source: SHP Single Base Map Data) 
Borax use has been prohibited by the Minister of Health through a regulation statement issued called as SK (Decree) No. 722/MENKES/PER/IX/88. Winarno (1997) states that borax toxicity stated in acute $\mathrm{LD}_{50}$ (lethal dose) is $4.5-4.98 \mathrm{~g} / \mathrm{kg}$ of mice body weight. Consumed borax will be absorbed by the body and stored cumulatively in the liver, brain, and testicles (Winarno, 1997). A high dose of borax in the body can cause nausea, throw up, diarrhea, stomach cramp, and the acute lethal dose for an adult is 10-20 grams or more (US Department of Health and Human Services, 2010).

Based on Etimine USA, Inc., Safety Data Sheet, oral $\mathrm{LD}_{50}$ is $3500 \mathrm{mg} / \mathrm{kg}-4100$ $\mathrm{mg} / \mathrm{kg}$ of mouse weight. Dermal LD is 2000 $\mathrm{mg} / \mathrm{kg}$ of rabbit weight. Inhale $\mathrm{LC}_{50}$ (lethal concentration) of borax acid is $>2.03 \mathrm{mg} / \mathrm{L}$ of mouse weight for 4 hours. Meanwhile, Saparinto et al. (2006) state that the highest borax dosage is $10 \mathrm{~g} / \mathrm{kg}$ of BW-20 g/kg adult $\mathrm{BW}$ and $5 \mathrm{~g} / \mathrm{kg}$ of children BW, which causes poisoning and death. Based on the toxicity data, the research result is still far from toxicity dosage. However, the fact that meatball skewer is an inexpensive and frequently consumed food raises concern that it is accumulated in the body and is dangerous. There should be strong supervision from various parties such as the government, people, and vendors who do not use the dangerously addictive substance so that the foods sold have good quality and are worth consuming.

\section{CONCLUSION}

Based on the survey analysis with 34 samples taken in 17 sub-districts, all meatball skewers sold in Bantul District, Special Region of Yogyakarta contain borax with concentration ranged between $0.06 \%$ $5.15 \%$.

\section{AKNOWLEDGEMENT}

Financial and facilities support from Universitas Muhammadiyah Yogyakarta to the authors are highly acknowledged.

\section{REFERENCES}

Aryani, T., Widyantara, A.B. 2018. Analisis Kandungan Boraks Pada Makanan Olahan Yang Dipasarkan di Sekitar Kampus. Jurnal Riset Kesehatan, 7(2), $106-109$.

Asteriani, Elmatris, Endrinaldi. 2008. Identifikasi dan Penetapan Kadar Boraks pada Mie Basah yang Beredar Dibeberapa Pasar di Kota Padang. Majalah Kedokteran Andalas, 32(2), $174-179$.

BPS Kabupaten Bantul. 2015. Luas Wilayah dan Banyaknya Desa Menurut Kecamatan di Kabupaten Bantul, Bantul: Badan Pusat Statistik.

Clarke, E. G. C., Moffat, A.C., Osselton, M. D., Widdop, B. 2004. Clarke's Analysis of Drugs and Poisons. London: Pharmaceutical Press.

Day, J.R.R.A., Underwood, A.L. 1996. Analisis Kimia Kuantitatif, edisi ke 6. Jakarta: Penerbit Erlangga.

Department of Health and Human Services of the United States. 2010. Toxicological Profile for Boron. Atlanta: Agency for Toxic Substances and Disease Registry: Division of Toxicology and Environmental Medicine/Applied Toxicology Branch.

Grynkiewicz, G., Ślifirski, P. 2012. Curcumin and curcuminoids in quest for medicinal status. Acta Biochimica Polonica, 59(2)

Harimurti, S., Setiyawan, A. 2019. Analisis Kualitatif dan Kuantitatif Kandungan Boraks Pada Bakso Tusuk di Wilayah Kabupaten Gunungkidul Provinsi Daerah Istimewa Yogyakarta. Farmasains: Jurnal Ilmiah Ilmu Kefarmasian, 6(2), 43-50.

Nursalam, 2013. Konsep Dan Penerapan Metodologi Penelitian Ilmu Keperawatan: Pendekatan Praktis. Edisi 3. Salemba Medika. Jakarta.

Rahmat, S., Tamrin, Ibrahim, M.N. 2017. Pengaruh Penambahan Kitosan Dan Lama Penyimpanan Bakso Ikan Tongkol (Euthynnus Affinis C.) 
Terhadap Nilai Organoleptik, Kadar Air Dan Jumlah Bakteri. J. Sains dan Teknologi Pangan, 2(2), 444 - 457.

Roth, H.J., Blaschke, G. 1998. Analisis Farmasi. Airlangga University Press. Surabaya.

Saparinto, C., Hidayati, D. 2006. Bahan Tambahan Pangan. Kanisius. Yogyakarta

The Minister of Health of The Republic of Indonesia, 1988. Regulation No. 722/MENKES/PER/IX/88, Food Additives. Jakarta.
The Minister of Health of The Republic of Indonesia. 1999. Regulation No. 1168/MENKES/PER/X/1999, Food Additives. Jakarta

Tubagus, I., Citraningtyas, G., Fatimawali, 2013. Identifikasi dan Penetapan Kadar Boraks Dalam Bakso Jajanan di Kota Manado. PHARMACON Jurnal Ilmiah Farmasi - UNSRAT, 2(4), 142 - 148.

Wibowo, S. 2005. Pembuatan Bakso Daging dan Bakso Ikan. Jakarta: Penebar Swadaya.

Winarno, F.G. 1997. Keamanan Pangan. Institut Pertanian Bogor. Bogor 